\title{
HoxD cluster scanning deletions identify multiple defects leading to paralysis in the mouse mutant Ironside
}

\author{
Basile Tarchini, ${ }^{1}$ Thi Hanh Nguyen Huynh, ${ }^{1}$ Greg A. Cox,${ }^{2}$ and Denis Duboule ${ }^{1,3}$ \\ ${ }^{1}$ National Research Centre 'Frontiers in Genetics' and Department of Zoology and Animal Biology, University of Geneva, \\ 1211 Geneva 4, Switzerland; ${ }^{2}$ The Jackson Laboratory, Bar Harbor, Maine 04609, USA
}

\begin{abstract}
A spontaneous semidominant mutation (Ironside, Irn) was isolated in mice, leading to severe hindlimb paralysis following multiple deletions in cis at the $\operatorname{HoxD}$ locus. To understand its cellular and molecular etiology, we embarked on a comparative analysis using systematic $\operatorname{Hox} D$ cluster deletions, produced via targeted meiotic recombination (TAMERE). Different lines of mice were classified according to the severity of their paralyses, and subsequent analyses revealed that multiple causative factors were involved, alone or in combination, in the occurrence of this pathology. Among them are the loss of Hoxd10 function, the sum of remaining Hoxd gene activity, and the ectopic gain of function of the neighboring gene $E_{v x} 2$, all contributing to the mispositioning, the absence, or misidentification of specific lumbo-sacral pools of motoneurons, nerve root homeosis, and hindlimb innervation defects. These results highlight the importance of a systematic approach when studying such clustered gene families, and give insights into the function and regulation of Hox and Evx2 genes during early spinal cord development.
\end{abstract}

[Keywords: Homeosis; peroneal nerve; chromosome engineering; motoneuron]

Supplemental material is available at http://www.genesdev.org.

Received May 11, 2005; revised version accepted October 3, 2005.

Mammalian genomes contain 39 Hox genes, organized in four clusters; HoxA to HoxD (McGinnis and Krumlauf 1992). These series of contiguous genes encode transcription factors necessary for proper development to proceed. Coordinate expression of Hox genes, in both time and space, is partly dictated by their order along the chromosome, such that genes are activated one after the other, from the $3^{\prime}$-end of the gene cluster toward its $5^{\prime}$-extremity. Genes activated early on will establish an anterior expression boundary in a variety of trunk derivatives, whereas subsequent activations will generate progressively more posterior boundaries, following molecular mechanisms that only begin to be understood (Kmita and Duboule 2003).

Collinear Hox gene expression is found in many developing systems, such as in paraxial mesoderm (Gaunt et al. 1988), in the digestive and the uro-genital tracts (Dollé et al. 1991; Yokouchi et al. 1995), or in budding limbs (Dollé et al. 1991). It is also observed in the embryonic spinal cord and hindbrain, where Hox genes are characterized by a precise rostral expression boundary

${ }^{3}$ Corresponding author.

E-MAIL Denis.Duboule@zoo.unige.ch; FAX 41-22-379-6795.

Article and publication are at http://www.genesdev.org/cgi/doi/10.1101/ gad.351105.
(Gaunt et al. 1988). The function of these genes during the development of the central nervous system (CNS) has been addressed in the presumptive rhombencephalon, due to the existence of morphological and molecular landmarks. The importance of Hox gene products in both the segmental organization of hindbrain neurons (Lumsden and Krumlauf 1996) and neural crest derivatives (Trainor and Krumlauf 2001) was thus demonstrated.

Yet few studies have addressed the function of Hox genes in the spinal cord, due to both the difficulty of assigning morphological landmarks and the functional redundancy of the Hox system (Carpenter 2002). Consequently, while Hox gene loss-of-function approaches lead to alterations in vertebral morphologies, neural alterations at comparable body levels are more difficult to document, in particular outside the limb territories, which seem to require more complex Hox-associated patterning information such as that necessary to organize finer topological distinctions. Accordingly, roles for these genes in the spinal cord have been mostly linked to the brachial and lumbo-sacral plexii, innervating the fore- and hindlimbs, respectively. For example, mice lacking Hoxc8 function display an aberrant forelimb connectivity pattern (Tiret et al. 1998), and Hoxd10 disruption resulted in hindlimb gait and position defects 
associated with reduction in both the peroneal nerve and the terminal arborization of the tibial nerve, suggesting that alterations in motoneuron ( $\mathrm{MN}$ ) positional identities impacted on the peripheral innervation pattern (Carpenter et al. 1997; Wahba et al. 2001).

Hox gene expression in the spinal cord is dynamic, often starting in broad ventricular regions, before postmitotic neural commitment occurs, and subsequently maintained in some MN or interneuron (IN) subtypes. Interestingly, paralogous genes from different clusters have related AP expression domains but may respond to distinct dorsoventral patterning signals (Graham et al. 1991; Davenne et al. 1999). Recently, a functional relationship between Hox expression and MN specification was documented as HOXC6 and HOXC9 protein expression coincided with particular brachial and thoracic motoneurons (Dasen et al. 2003). Ectopic expression of either protein induced both the extinction of the other and a concomitant change in MN subtype. Therefore, HOX cross-repressive interactions may establish distinct rostro-caudal MN fate as well as MN pool identity within a given columnar subtype (Dasen et al. 2003). Likewise, Hoxd10 could specify the lateral motor column (LMC) at lumbar levels (Shah et al. 2004).

A mouse with a severe semidominant hindlimb paralysis was discovered, which carried a spontaneous mutation allelic to the HoxD cluster. To understand why this phenotype was more severe than that induced by a targeted deletion of the entire cluster (Zakany et al. 2001) and gain insights into the role of Hox genes in hindlimb motricity, we engineered a set of targeted deletions (TAMERE) (Hérault et al. 1998). The analysis of various deleted strains and the new mutant stock allowed us to distinguish between different categories of phenotypes and to correlate these categories with distinct defects in MN specification and axonal projection at lumbo-sacral levels. This systematic approach shed light on the function of Hox genes in the specification of $M N$ and identified the ectopic expression of Evx2 as the cause of the most severe phenotype, in addition to the loss of Hoxd10 function.

\section{Results}

\section{The Ironside mutation is allelic to HoxD}

A mouse with a spontaneous mutation was isolated due to severe hindlimb paralysis (Fig. 1A). Further crosses narrowed down the mutated locus to a 6-cM interval between D2Mit10 and D2Mit418 on chromosome 2. Heterozygous mice were bred to homozygosity, which produced an even more severe condition with complete hindlimb paralysis as well as digit malformations. Recessive digit shortening suggested that Hoxd genes, located within the interval, were involved (Dollé et al. 1993), and subsequent PCR amplification indicated that mutant mice had lost Hoxd13 to Hoxd8, whereas the neighboring Evx2 and Hoxd4 genes were intact.

The cloning of the breakpoints revealed a double deletion in cis, separated by a short piece of DNA. The first deletion removed a 43-kb large fragment containing from Hoxd13 to Hoxd9, whereas the second contained an 8-kb large fragment including the second exon of Hoxd8 and 3 ' sequences (Fig. 1B). The two deletions were separated by the first exon of Hoxd8 and part of its 5'-UTR and intronic sequences, linked to a 200-base-pair (bp) fragment coming from an inverted duplication of a noncoding locus located $394 \mathrm{~kb}$ centromeric to the Evx2 gene.
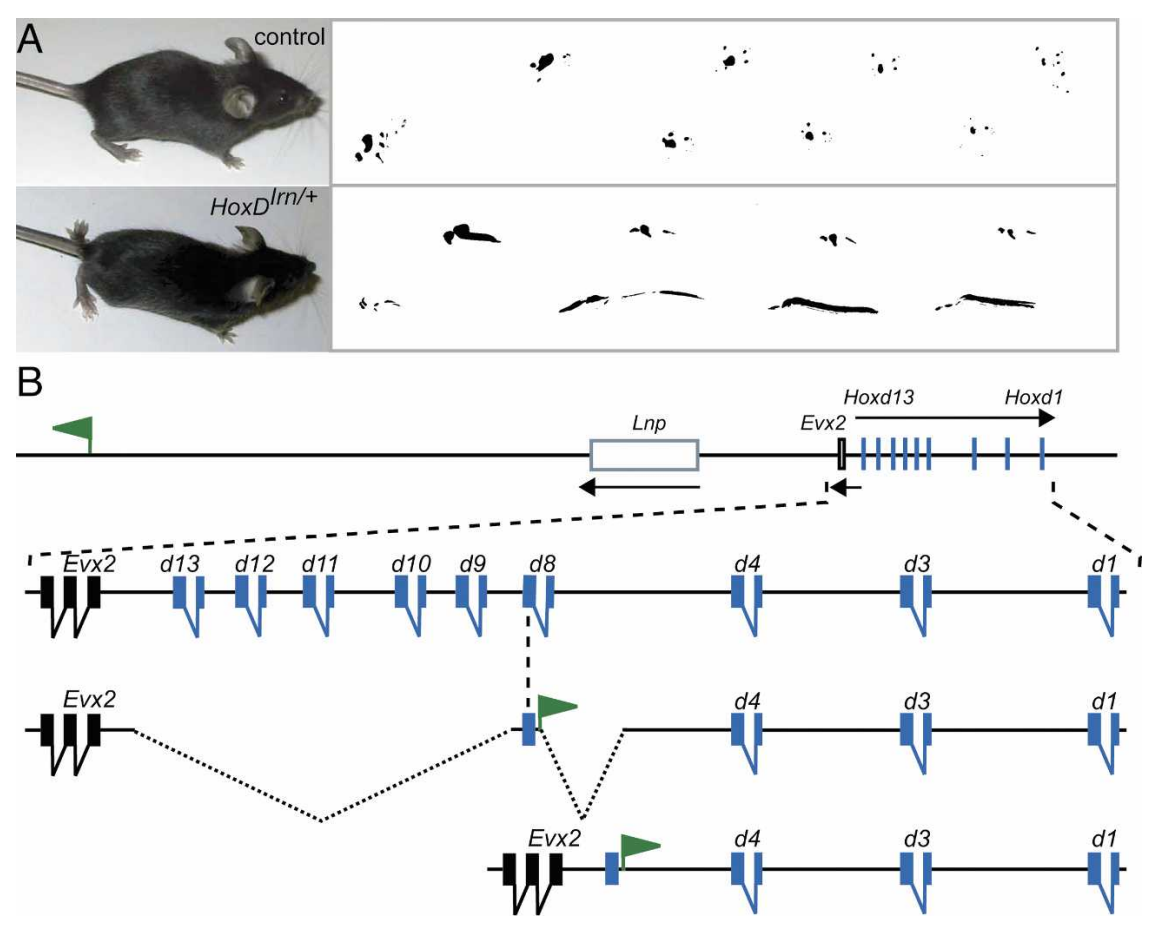

Figure 1. The spontaneous mutation Ironside (Irn). ( $A$, bottom) Mice heterozygous for Irn $\left(\operatorname{Hox}^{\operatorname{Irn} /+}\right)$ display severe hindlimb paralysis, as illustrated by footprint analyses. $(B)$ The Irn mutation is a combined deletion/insertion event at the HoxD cluster. Hox genes are indicated in blue, whereas Evx2 is in black. The mutated locus contains two deletions in cis (dashed lines) removing from Hoxd13 to within Hoxd8, and from within Hoxd8 further 3'. A fragment of Hoxd8 was left in place (bottom line; blue box), along with a piece of DNA translocated from a region located centromeric to the locus (top and bottom lines; green flag). This latter, noncoding sequence is still present at its original locus in the mutant configuration, in an inverted orientation. No other genomic rearrangement was detected outside the HoxD cluster. 
Tarchini et al.

This latter locus appeared nevertheless intact in mutant mice. This mutant stock, called Ironside (Irn), is therefore an allele of the HoxD cluster [Del(2Hoxd8,Hoxd9Hoxd13)], referred to below as HoxD ${ }^{\operatorname{Irn}}$ or Irn.

\section{TAMERE-induced deletions reveal classes of paralyzed phenotypes}

To understand the molecular etiology of this severe phenotype, we produced four mouse strains carrying a Lox $P$ site targeted between selected pairs of Hoxd genes (Fig. 2, L5, L6, L7, L8). These mice, as well as others previously available (Fig. 2, L1, L4, L11), were intercrossed to induce unequal meiotic recombinations, leading to the deleted strains depicted in Figure 2. We selected a set of deletions resembling the Irn mutation, that is, starting upstream of Hoxd13. We also analyzed smaller internal de- letions, with or without Hoxd10, as loss of function of this gene induced mild hindlimb defects (Carpenter et al. 1997). These deletion stocks are referred to as, for example, Del(10-13), for the Hox $D^{\operatorname{Del}(10-13)}$ allele (a deletion removing from Hoxd10 to Hoxd13). One deletion was associated with the cis inactivation of the neighboring gene Evx2 [Del(10-13); Evx2 $\left.2^{\text {stop }}\right]$. The intergenic region between Hoxd4 and Hoxd8, flanked by the L7 and L8 $\operatorname{lox} P$ sites, was named region "i."

This collection of mutant animals revealed a range of defects in locomotion and posture. Complete hindlimb paralysis was scored in specimens homozygous for three different deletions, all with a 5' breakpoint between Evx2 and Hoxd13 [Fig. 2, Del(10-13), Del(9-13), and Del(i-13)]. The defects were similar to those in the Irn mutant, confirming that the HoxD rearrangement was the cause of the Irn paralysis. These three deletions and

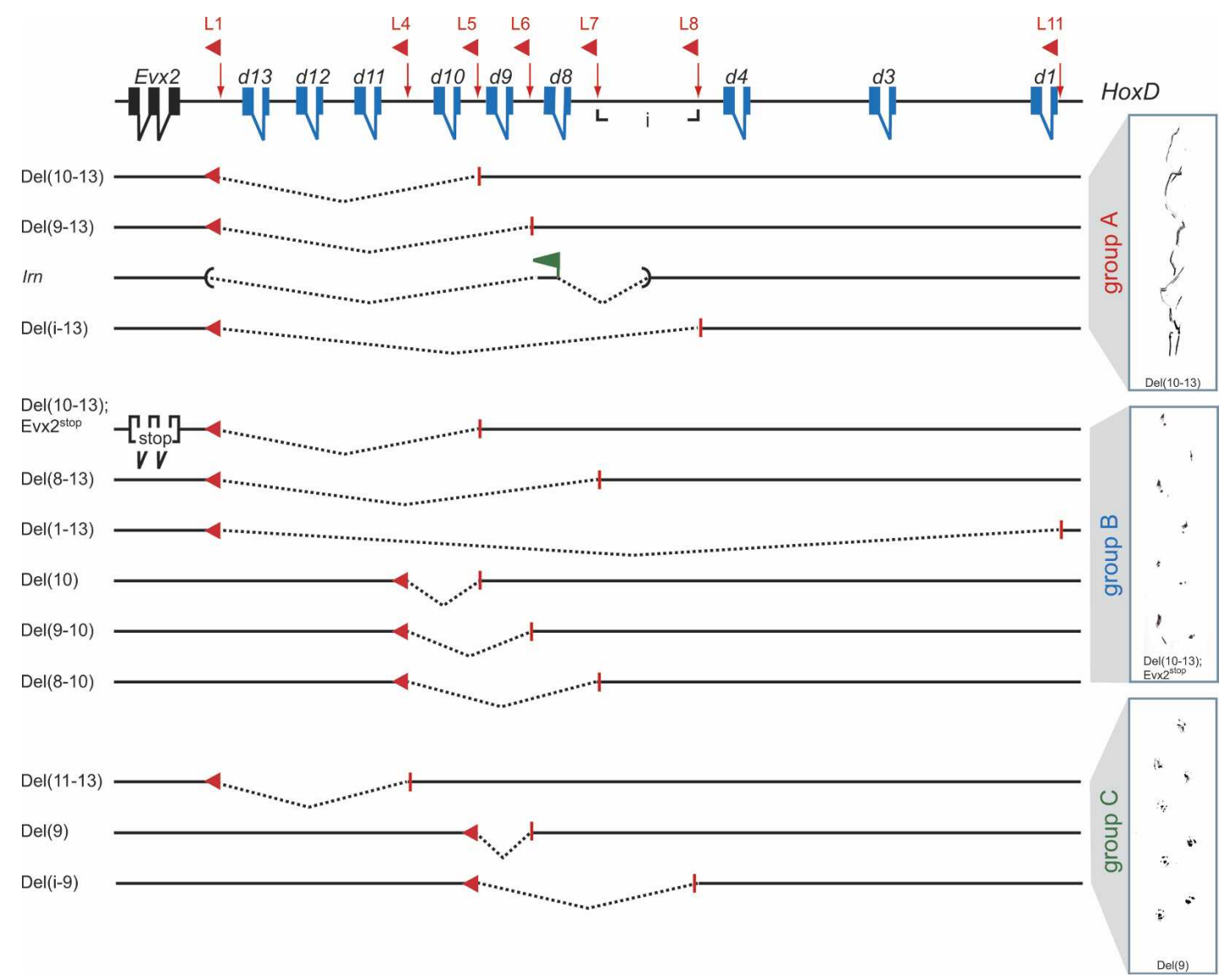

Figure 2. Scheme of deleted mouse strains used in this work. On the top, the HoxD cluster with the position of the various LoxP sites (red). Each site represents an independent mouse strain. The strains containing the L5, L6, L7, and L8 sites were produced for this study, whereas others were described previously. Below are 11 deleted strains generated by recombinations between pairs of Lox $P$ parental lines using the technique TAMERE (Hérault et al. 1998). The Ironside (Irn) and Del(1-13) alleles are also included. Breakpoints are in red, with a triangle for the remaining $L o x P$ site. The deleted fragments are shown in dashed lines. For convenience, the strains are referred to as, for example, Del(10-13) for a deletion removing from Hoxd10 to Hoxd13, inclusively [HoxD Del(10-13) . One configuration carries in addition a stop codon engineered within the neighboring Evx2 gene $\left(E_{V x} 2^{\text {stop }}\right)$. These deleted strains are classified in three groups, with the same color code throughout the paper, based on the severity of the paralysis, documented by footprint analyses of representative homozygous specimen. Group A (red) deletions are dominant and lead to complete paralysis, whereas group B (blue) animals have a recessive and less severe phenotype. Group C (green) animals have no obvious hindleg problem. The inactivation of Evx2 in the Del(10-13) deletion significantly rescues the locomotion defect. 
the Irn allele also shared a semidominant phenotype and were referred to as "group A" alleles (Fig. 2).

A second set of deletions (Fig. 2, Group B) showed more heterogeneous phenotypes, less severe than in group A, with recessive locomotion defects due to a distally restricted leg paralysis, even though they shared with group A their 5' breakpoint [Fig. 2, Del(10-13); $E_{v \times} 2^{\text {stop }}$ and $\left.\operatorname{Del}(8-13)\right]$. Animals of this group could bear their body weight on the hindlegs and retained left-right alternation in walking (Fig. 2, cf. footprints). Foot placement and gait were reminiscent of clubfoot phenotypes (e.g., Helmbacher et al. 2000). Interestingly, the full HoxD deficiency (Zakany et al. 2001) fell into this group, as well as internal deletions lacking the Hoxd10 gene [Fig. 2, Del(10), Del(9-10), and Del(8-10)]. Finally, a third class of strains (Fig. 2, group C) displayed apparently normal locomotion and posture. These included one stock with a breakpoint at the L1 position [Fig. 2, Del(11-13)], as well as internal deletions [Fig. 2, Del(9), Del(i-9)], all of them leaving Hoxd10 intact.

\section{Peroneal nerve defects in HoxD deletions}

These deleted strains showed no detectable alteration in embryonic limb muscle patterning. Instead, striking defects were observed in the peroneal nerve branch of mutant fetuses. This branch emanates from the dorso-sacral hindlimb plexus and targets various muscles, among which are the extensors of the leg latero-distal compartment. Anti-neurofilament immunostaining revealed a range of peroneal defects, already visible at day 12.5. Peroneal nerve branches were classified as "close to normal," "thinner and/or shorter," "truncated," or "absent" (Fig. 3A). In the former category, the growth of peroneal branches was comparable to that of the adjacent tibial branch. While thinner and/or shorter peroneal branches entered the limb bud, they contained fewer fibers and/or showed reduced or delayed distal progression. Truncated branches displayed a nerve trunk past the plexus region, but few fibers had entered the limb bud proper at embryonic day 13.5 (E13.5). The "absent" category referred to a
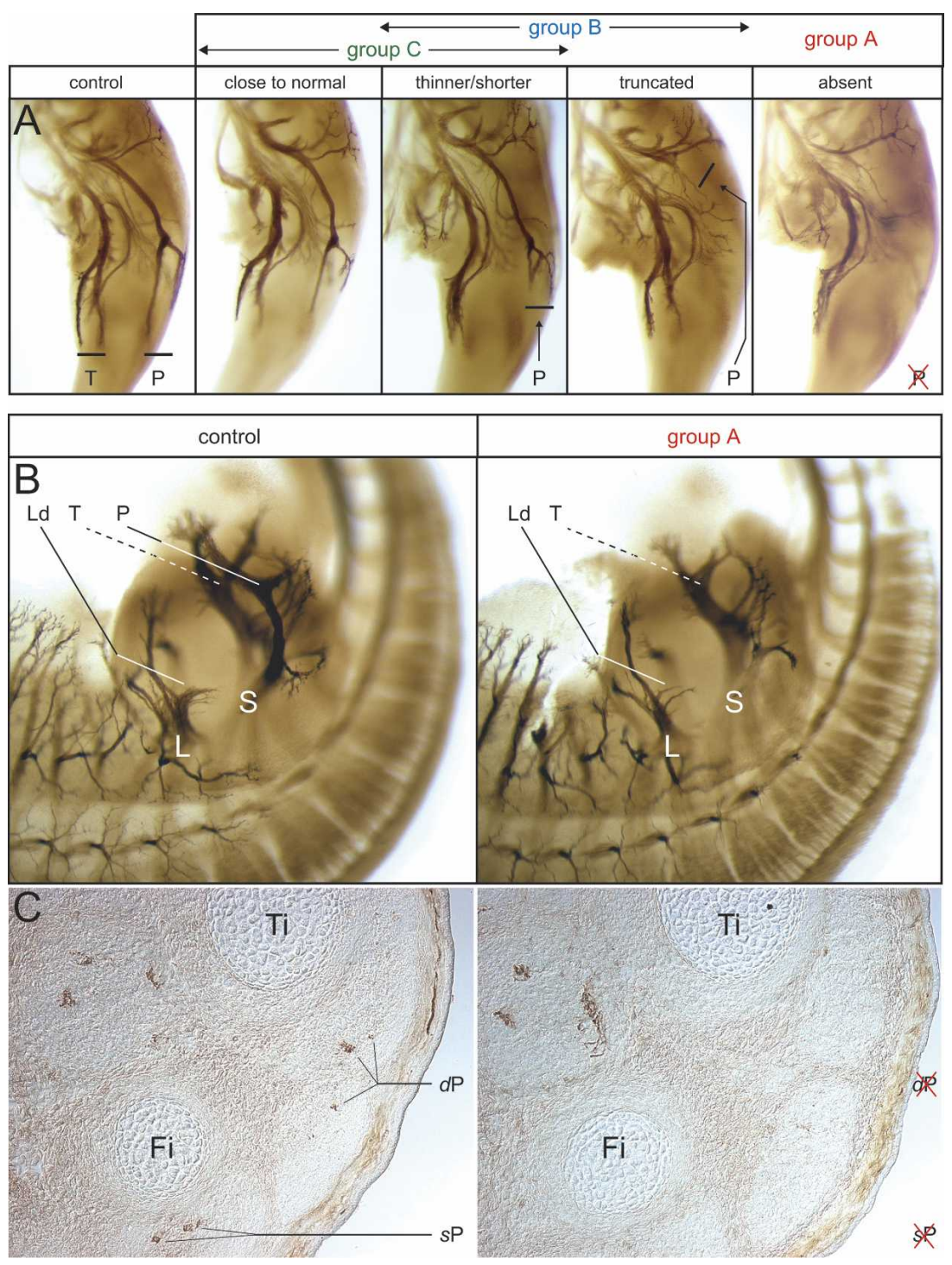

Figure 3. Peroneal nerve defects in HoxD deletion strains. (A) Whole-mount neurofilament immunostainings of E12.5-E13.5 hindlimbs (posterior view, dorsal is right and distal at the bottom) differentiate various peroneal nerves as "close to normal," "thinner/shorter," and "truncated" or "absent." Group C animals show close to normal or thinner/shorter peroneal nerves; group B animals display thinner/shorter or truncated phenotype, whereas severely affected group A animals show no peroneal nerve. $(B$, right) In addition to the complete loss of the dorsosacral plexus and emanating peroneal nerve $(\mathrm{P})$, group A mutants also show innervation defects in the dorso-lumbar plexus (Ld), where fewer fibers are visible. Pictures are lateral views of a right hindlimb. (C) E14.5 leg crosssections at shank level stained for neurofilaments revealed the absence of the deep (dP) and superficial $(\mathrm{sP})$ peroneal nerves in group $\mathrm{A}$ specimens. ( $\mathrm{T}$ ) Tibial nerve (ventral); (P) peroneal nerve (dorsal); (L) lumbar plexus (anterior); (S) sacral plexus (posterior); (Fi) fibula, (Ti) tibia. 
very thin peroneal nerve, likely consisting of sensory components only.

While group $\mathrm{C}$ animals showed close to normal or thinner/shorter peroneal branches (Fig. 3A), group B alleles led to variable proportions of thinner/shorter versus truncated branches. Expectedly, peroneal branches were absent in group A animals (Fig. 3A,B), and cross-sections of E14.5 hindlimbs at shank level confirmed the loss of the peroneal nerves (Fig. 3C). Dissection of adult legs revealed the absence of the common peroneal nerve with full or partial penetrance in group A and B animals, respectively. Furthermore, a severe atrophy of the leg antero-lateral compartment muscles was observed in group A animals, suggesting subsequent degeneration. This tight correlation with the severity of the paralysis indicated that peroneal innervation defects play a major role in the phenotype. Neurofilament staining of group A embryos also revealed other peripheral nerve defects such as a reduced dorso-lumbar plexus (Fig. 3B). The tibial branch, originating from the ventrosacral plexus, appeared normal in all deleted stocks (Fig. 3A,B).

\section{Nerve roots homeosis}

As innervation deficits can arise from alterations in either peripheral navigation, or the neuronal specification in the spinal cord, we looked at lumbo-sacral (LS) spinal root morphology in the various deletions and scored several defects. LS spinal roots have distinct migratory trajectories, which confer their unique identities. In wildtype embryos, the 21 st-23rd roots contribute to the lumbar plexus, whereas the 23rd-25th contribute to the sacral (sciatic) plexus (Fig. 5A, below). For each plexus, axonal fibers adopt either a ventral or a dorsal trajectory after entering the limb bud.

Homeotic transformations were observed in several deleted alleles. They were mostly recessive and changed a nerve root into the identity of its rostral neighbor, such that the LS root pattern appeared shifted posteriorly (anterior homeosis). Transformations were often partial and some LS nerve roots could be transformed, whereas others remained unchanged. For example, in the Del(10-13); Evx $2^{\text {stop }}$ allele, only the 25th and 26th roots appeared transformed into the 24th and 25th identities, respectively (Fig. 4C). Incomplete transformations with a mixed roots profile are illustrated by the Irn mutant, where, in addition to a full transformation of the 25 th and 26th roots, the 23rd and 24th roots appeared only partially transformed into $22 \mathrm{nd}$ and 23rd root identities, respectively (Fig. 4B). In contrast, Del(10) mutant mice displayed a complete and dominant posterior transformation, with all LS roots transformed into the identity of the next caudal neighbor (Fig. 4D). Consequently, the LS nerve pattern was normal yet displaced rostrally, leading to the loss of one thoracic root.

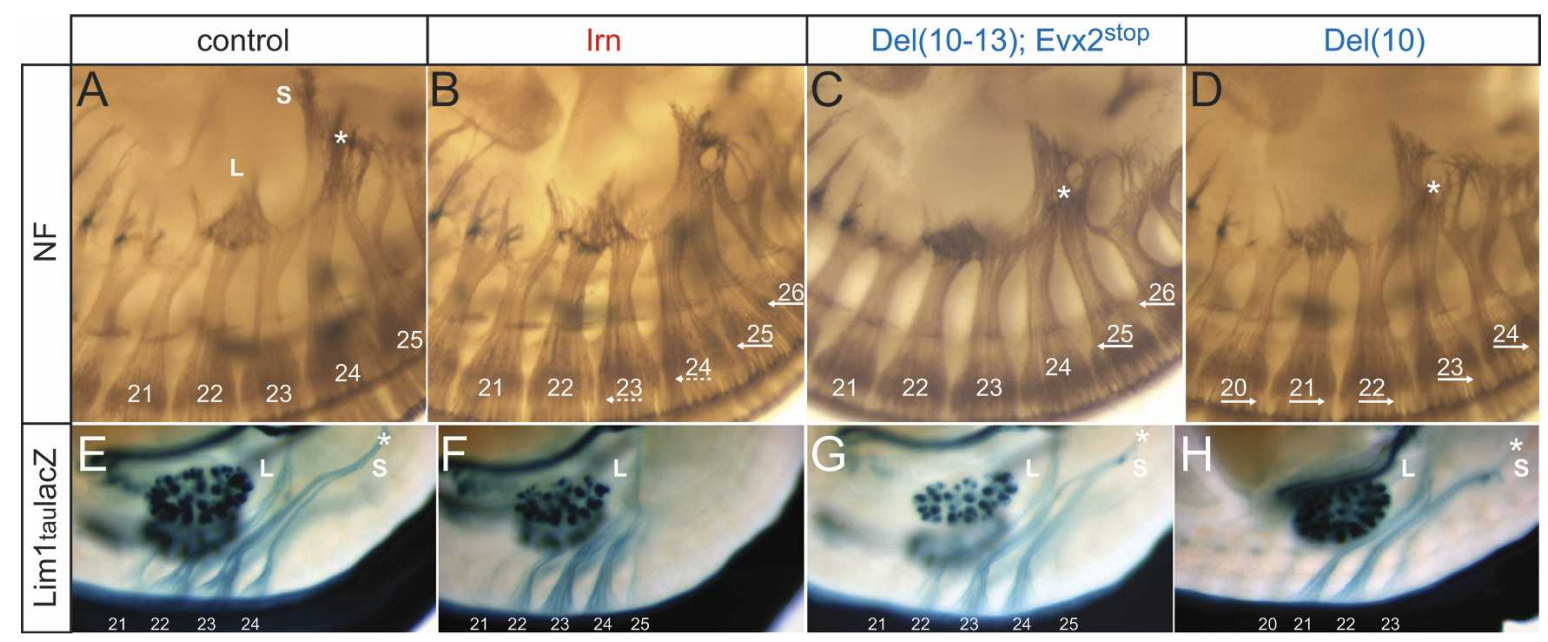

Figure 4. Homeotic transformations in HoxD deletion strains. (A) Neurofilament stainings at E11.5-E12.5 show that roots 21-23 participate in the lumbar plexus, whereas roots $23-25$ form the sacral plexus, with characteristic projection patterns. $(B)$ The 21 st and 22nd roots are unchanged in Irn mutants, whereas more caudal roots are transformed into anterior identities, with different magnitudes, such that, for example, the 25th and 26th mutant roots appear like normal 24th and 25th. $(C)$ Transformations are more restricted in Del(10-13); Evx $2^{\text {stop }}$ mutants, as only the caudal-most 25th and 26th roots appeared transformed. (D) In contrast, posterior transformations are seen in the $\operatorname{Del}(10)$ allele, with all LS roots looking like their immediate posterior neighbor. $(E)$ Crosses between HoxD deletions and a Lim1taulacZ knock-in transgenic line reveal the motor projections to the dorsal limb. Motor fibers in roots 21-24 are labeled, whereas only few (if any) fibers are detected in the 25th root. (F) The Irn mutant shows two motor defects: the absence of the dorso-sacral plexus (S) and anterior homeotic transformations. In this latter case, projections from the $23 \mathrm{rd}$ root mostly target the lumbar plexus, instead of going to both lumbar and sacral plexii, and the 25th root is stained, indicating a more anterior identity. $(G)$ In contrast, a dorso-sacral plexus is visible in Del(10-13); Evx2 ${ }^{\text {stop }}$ mutants, which have more subtle anterior homeosis, such as some positive fibers in the 25 th root. $(H)$ The Del(10) allele also shows a dorso-sacral plexus, whereas posterior homeosis transformed all motor nerves contributing to the hindlimb. Roots are numbered, with plain and dashed arrows indicating complete or partial transformation, respectively. Arrows to the left and right indicate anterior and posterior transformations, respectively. (L) Lumbar plexus; (S) sacral plexus. Asterisks indicate the dorso-sacral plexus and emanating peroneal branch. 
To discriminate between axonal guidance and spinal autonomous defects, we crossed selected deletion strains with mice carrying a tau-lacZ reporter knocked in the Lim1 gene (Kania et al. 2000). Lim1 is expressed in the lateral subset of LMC motoneurons, present only at limb levels. As all Lim1-positive MN send axons toward the dorsal limb, we visualized the dorsal motor nerve component in our mutant stocks. Control staining showed that only the $23 \mathrm{rd}$ and 24 th roots contribute to the dorsosacral plexus (Fig. 4E). As the 25th root axons navigate mostly toward the ventral aspect of the sacral plexus, staining was not scored at this level.

Irn embryos showed anterior transformations from the 21 st to the 25 th motor roots (Fig. 4F). Lim1-positive MN included an ectopic caudal population abnormally projecting through the 25 th root. These abnormal projections were reduced in the Del(10-13); Evx $2^{\text {stop }}$ allele, which showed a weaker homeotic phenotype in the 21 st-24th roots region (Fig. 4G). The Del(10) allele displayed global posterior motor nerve homeosis, as with neurofilament staining (Fig. $4 \mathrm{H}$ ). These results indicated that the homeosis derives from misspecification in the LS spinal cord, the anterior nerve homeosis reflecting a caudal shift in the Lim1-positive LMC. Prefiguring limb innervation deficits, group A specimens did not form the dorso-sacral plexus, from which the peroneal branch emerges, whereas group B alleles showed a starting peroneal branch (Fig. 4F,H). In this latter case, fibers must initially converge to the dorso-sacral plexus and initiate a peroneal branch, but further degenerate around the limb entry point. The inability to form a plexus thus correlates with strong motor homeosis in the LS region, as if transformed 24 th and 25 th roots were unable to endorse a normal $23 \mathrm{rd}$ and 24 th roots motor profile.

The progressive sequence of anterior homeotic transformations, as induced by the various deletions, is depicted in Figure 5. Homeosis is first seen caudally, suggesting a duplicated 24th root (Fig. 5D, control in C). Further transformations affect the $23 \mathrm{rd}$ root, which redirects progressively more axons toward the lumbar plexus at the expense of the sacral plexus (Fig. 5E). During this process, the 21 st root usually reduces its contribution to the lumbar plexus. At some point, the 23rd nerve exhibits a 22nd-like morphology, whereas the 22nd and 24th nerves seem to retain their normal identities. Therefore, root patterns suggest the duplication of the 22nd and 24 th nerves and, consequently, the absence of a 23rd mixed nerve. Complete transformations affect both the 22 nd and 24th roots, which thus adopt a 21 st and 23rd profile, respectively, as reported for the loss of function of Hoxa10 (Fig. 5F; Rijli et al. 1995). Misspecification of nerve trajectories was not necessarily coordinated between the ventral and dorsal aspects (data not shown).

In summary, all group A strains, as well as the majority of group B strains with a breakpoint upstream of Hoxd13, displayed partial 21st-24th and complete 25th and 26th roots anterior transformations (Fig. 5E). However, only group A showed severe motor homeosis using the Lim1taulacZ marker (Fig. 4F). In contrast, no consistent LS nerve misspecification was scored for group B internal deletions [e.g., $\operatorname{Del}(9-10) ; \operatorname{Del}(8-10)]$. Among group C strains, Del(11-13) showed a full transformation, but restricted to the 25 th and 26th roots (Fig. 5D), whereas Del(9) displayed partial 21st-24th and full 25th and 26th transformations (Fig. 5E). The Del(i-9) mice showed no homeosis. Since group B paralysis could occur without spinal root transformation and some transformations in group $\mathrm{C}$ had no effect on locomotion, we concluded that homeotic transformations are not a decisive factor in the locomotion defects. The specific partial transformation of the 23rd and 24th motor nerves was nevertheless associated with the strong phenotype of group A specimens.

\section{Spinal cord as the source of the phenotype}

These results suggested that alterations in LS nerves led to the observed paralysis. To rule out an impact of mutant hindlimb mesoderm on axonal navigation, we engineered a conditional strain carrying LoxP sites flanking the Hoxd13 and Hoxd4 genes [Fig. 6I, Flox(4-13)] and used the Prx1-Cre deleter transgene (Logan et al. 2002) to induce deletion in growing limbs only, without affecting the developing spinal cord. We looked at the effect in combination with the group A allele Del(10-13). Del(10 13) heterozygous animals often display a thinner and shorter peroneal nerve (Fig. 6A,B), which does not preclude a normal leg posture at birth. However, when a deletion of the Hoxd4 to Hoxd13 region [Del(4-13)] was added in trans, animals had severely truncated peroneal and bilateral clubfoot phenotypes (Fig. 6D). In contrast, when this latter deletion was induced only in developing limbs, by using the conditional allele, animals were mildly affected (Fig. 6C), with a peroneal branch resembling that of Del(10-13) heterozygous animals. We assessed the efficiency of the conditional deletion by looking at Hoxd10 transcripts in fetal limbs. In Del(10-13) heterozygotes, Hoxd10 was expressed in both the limbs and the trunk (Fig. 6A,B). While animals carrying both Del(10-13) and Del(4-13) alleles expectedly had no Hoxd10 signal, specimens carrying one copy of the conditional allele displayed a signal in the trunk, whereas limbs were devoid of Hoxd10 mRNAs (Fig. 6G). These results confirmed that the defects in the peroneal branch originate from alterations in the spinal cord, rather than in environmental cues necessary for axonal trajectories.

\section{Molecular bases of spinal cord homeosis}

Hox gene losses of function lead to anterior homeotic transformations. Furthermore, "posterior" Hox gene products are often functionally dominant over more "anterior" products (posterior prevalence) (Duboule and Morata 1994). In the LS region, Hoxd10 is the most posterior gene expressed between the 21st and 24th levels, until Hoxd11 starts to be transcribed at level 25. The former is thus expectedly an essential player for the hindlimb LMC region. Consistently, HoxD deletions starting upstream of Hoxd13 and extending up to at least Hoxd10 
showed anterior nerve root homeosis. However, internal deletions including Hoxd10 produced unexpected results; while both Del(9-10) and Del(8-10) showed a wildtype LS root organization, $\operatorname{Del}(10)$ had a complete posterior transformation and $\operatorname{Del}(9)$ displayed an anterior transformation, as for larger deletions where both Hoxd11 and Hoxd10 are missing.

To understand the molecular bases of these unexpected transformations, we analyzed the expression of the remaining Hoxd genes in all configurations. While Hoxd10, Hoxd9, Hoxd8, Hoxd4, and Hoxd3 are normally expressed at the LS levels, Hoxd11 is expressed caudal to the 25th level, and the expression limit of Hoxd12 and Hoxd13 is more caudal, making these genes irrelevant in this context. In $\operatorname{Del}(9)$, transcription of both Hoxd10 and Hoxd11 was shifted posteriorly, leading to anterior homeosis (Fig. 7A,B). Conversely, expression of
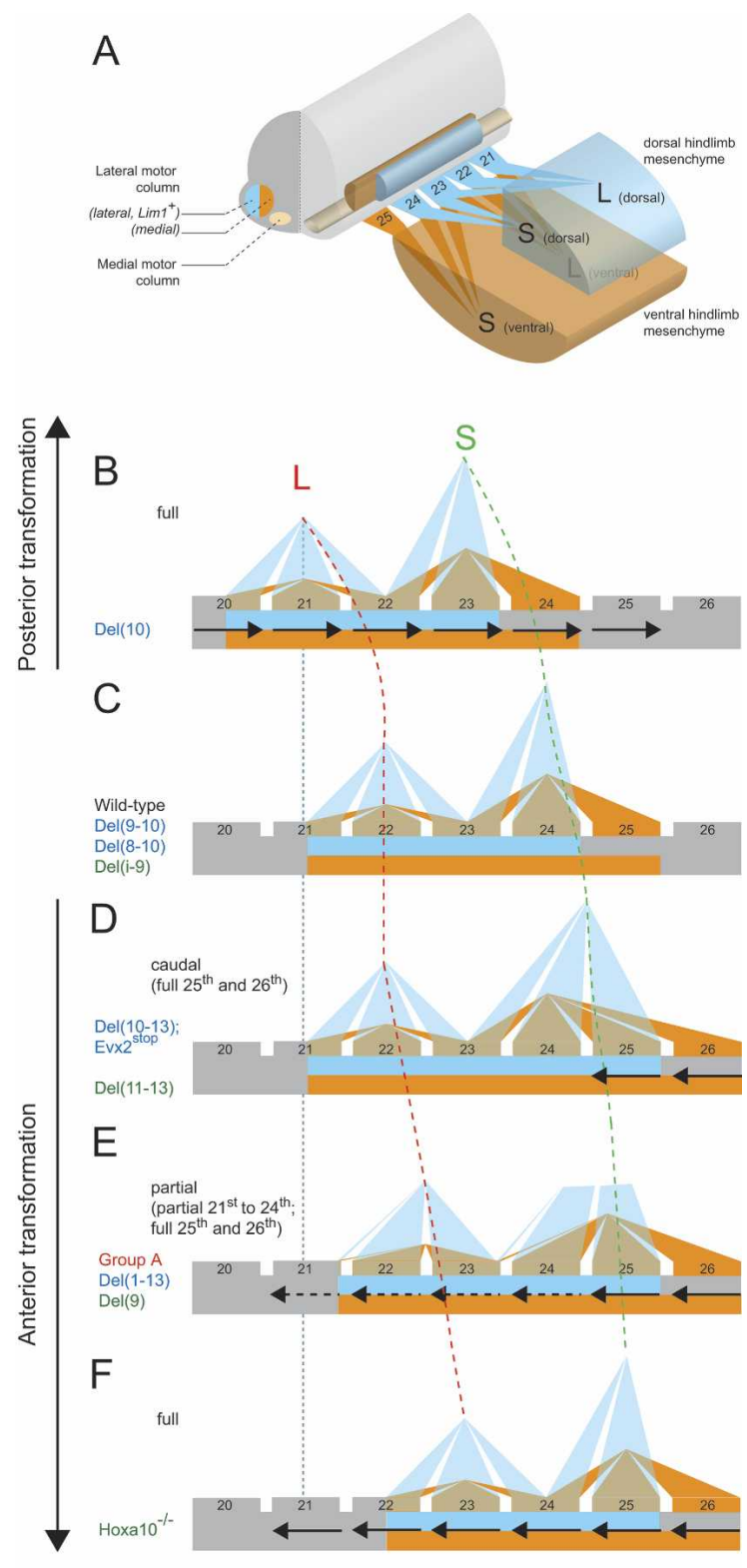

Hoxd11 in Del(10) was shifted anteriorly (Fig. 7C). In this latter case, the loss of Hoxd10 in lumbar spinal cord was more than rescued by the gain of function of Hoxd11, leading to a posterior transformation. Related anterior shifts of Hoxd11 were seen in both $\operatorname{Del}(9-10)$ and $\operatorname{Del}(8-$ 10) alleles, without transformation, likely due to the additional losses of function of Hoxd9 and Hoxd8 (Fig. 7D).

This correlation between Hoxd gene expression and patterns of homeotic transformations could nevertheless not account for all aspects of the paralysis phenotypes, in particular the difference between group A and B strains. As the full HoxD cluster deletion showed a mild group B paralysis, the severity of group A phenotypes could not be explained by multiple losses of function, nor by the observed Hoxd genes' gains of function. Since these latter deletions share a breakpoint upstream Hoxd13 (Fig. 2), near the $E_{V x} 2$ gene, we looked at Evx2 expression in various deletions.

\section{Evx2 misexpression in group A mutants}

Due to the presence of specific enhancers (Spitz et al. 2003) and a HoxD insulating mechanism (Kmita et al. 2002), Evx2 is normally expressed in specific interneurons throughout the developing spinal cord, that is, with a pattern distinct from Hoxd genes (Dollé et al. 1994; Moran-Rivard et al. 2001). In Irn embryos, we observed a dramatic gain of Evx2 expression along the entire spinal cord, encompassing virtually all cell types, resembling the neighboring gene Hoxd4 (Fig. 8A). Analysis of the three other group A alleles, Del(10-13), Del(9-13), and Del(i-13), confirmed this gain of expression, yet with some restriction to the ventral LS region (Fig. 8B; data not shown).

Figure 5. Sequence of progressive homeotic transformations, from a full segment posterior transformation $(B)$ to a full segment anterior transformation $(F)$. (A) Normal hindlimb bud innervation. LMC, nerve roots, plexii, and approximate projections are indicated with a color code used throughout the figure. (B) Posterior homeosis observed in Del(10). LS roots are fully transformed toward a posterior projection pattern. $(C)$ The wildtype organization is retained in some internal deletions [Del(i9); $\operatorname{Del}(8-10) ; \operatorname{Del}(9-10)]$. (D) Complete anterior transformations, but restricted to the 25th and 26th roots, are scored in group B Del(10-13); Evx2 $2^{\text {stop }}$ and Del(11-13) deletions. In this case, the LMC is caudally extended by one segment such that an additional root contributes to the sacral plexus. (E) More severe transformations also affect roots $21-24$, to various degrees. The latter roots are nevertheless only partially transformed. For instance, partial transformation of the 23rd root involves rerouting of its axonal projection toward the lumbar plexus, at the expense of the sacral plexus, leading to a root with a hybrid profile between the 22nd and 23rd aspect [group A or Del(1-13) and Del(9) alleles]. (F) Full transformation of all lumbar roots following Hoxa10 inactivation (Rijli et al. 1995), pointing to a full-segment caudal shift of the LMC. Lateral and medial subsets of the LMC are shown in blue and orange, respectively. Arrows to the right indicate segment and projection transformation toward a posterior fate, whereas arrows to the left indicate the converse transformation. 


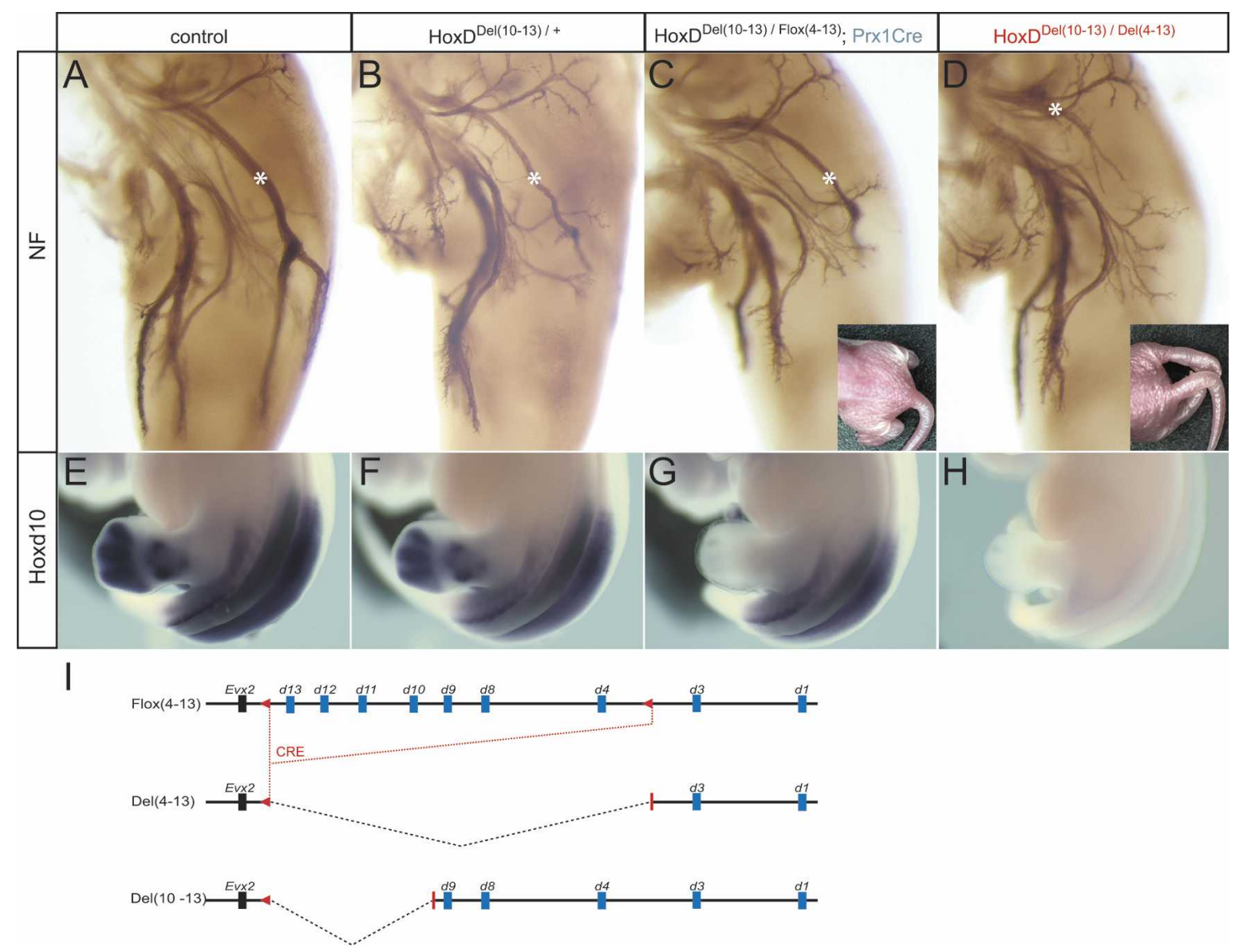

Figure 6. Innervation defects are spinal cord-autonomous. $(A-D)$ The floxed HoxD allele $[$ Flox $(4-13)$; depicted under $I]$ and the Prx1-Cre deleter transgene were used to conditionally delete Hoxd4-Hoxd13 in early developing limb tissues. (B, asterisk) Heterozygous Del(10-13) animals often showed a severe peroneal nerve reduction. $(B, C)$ Trans-heterozygous specimens carrying both the Del(10-13) and Flox(4-13) alleles along with the Prx1-Cre showed similar peroneal defects, yet with a normal leg posture at birth. $(C, D)$ In contrast, fully deleted trans-heterozygous animals [Del(10-13)/Del(4-13)] showed the expected truncated peroneal nerve and bilateral clubfoot at birth. $(G)$ The conditional deletion was monitored by the selective loss of Hoxd10 expression in E12.5 hindlimb buds.

In contrast, group B alleles sharing the same 5' breakpoint [Del(8-13) or Del(1-13)] displayed a normal Evx2 pattern in the spinal cord, as did group C Del(11-13) (Fig. 8B; data not shown). The impact of Evx2 on the severity of the phenotype was confirmed by the comparison between the $\operatorname{Del}(10-13)$ and $\operatorname{Del}(10-13) ; E_{v x} 2^{\text {stop }}$ alleles. Although these two alleles have the same deletion and a gain of Evx2 transcripts in the LS spinal cord, only the former belongs to group A, whereas the latter is in group $\mathrm{B}$, demonstrating that a functional EVX2 protein was required for a severe paralysis to occur.

We further engineered a transgenic strain carrying a BAC clone covering the HoxD cluster and containing both an Irn-like targeted deletion and a lacZ cassette in frame within Evx2 (Fig. 8C). Transgenic mice consistently reproduced the ectopic activation of Evx2 in the developing spinal cord (Fig. 8D). Furthermore, two distinct lines showed staining in the tibial and peroneal nerve branches emanating from the sacral plexus (Fig. $8 \mathrm{D})$, indicating that the Evx2 spinal gain of expression had indeed occurred in LMC motoneurons, in particular in peroneal motoneurons.

\section{Misspecification of motoneuron pools}

To assess how ectopic EVX2 interferes with MN specification, we compared group A and B deletions using the Raldh2 and Pea3 markers. The former labels the entire LMC (Sockanathan and Jessell 1998), and a comparison between $\operatorname{Del}(10-13), \operatorname{Del}(10-13) ; E v x 2^{\text {stop }}$, and wild-type configurations revealed a posterior shift in the mutant patterns, coherent with the nerve root homeotic transformations. In addition, a marked decrease in signal intensity was scored in the Del(10-13) specimen, that is, when a genuine EVX2 protein was ectopically expressed. This effect was general, yet particularly obvious at dorsal root ganglia (DRG) level 21-22 (Fig. 9A).

At hindlimb level, Pea3 is expressed by subsets of motoneuron pools within the LMC (Arber et al. 2000). In situ hybridization revealed the lumbar motor pools, with 


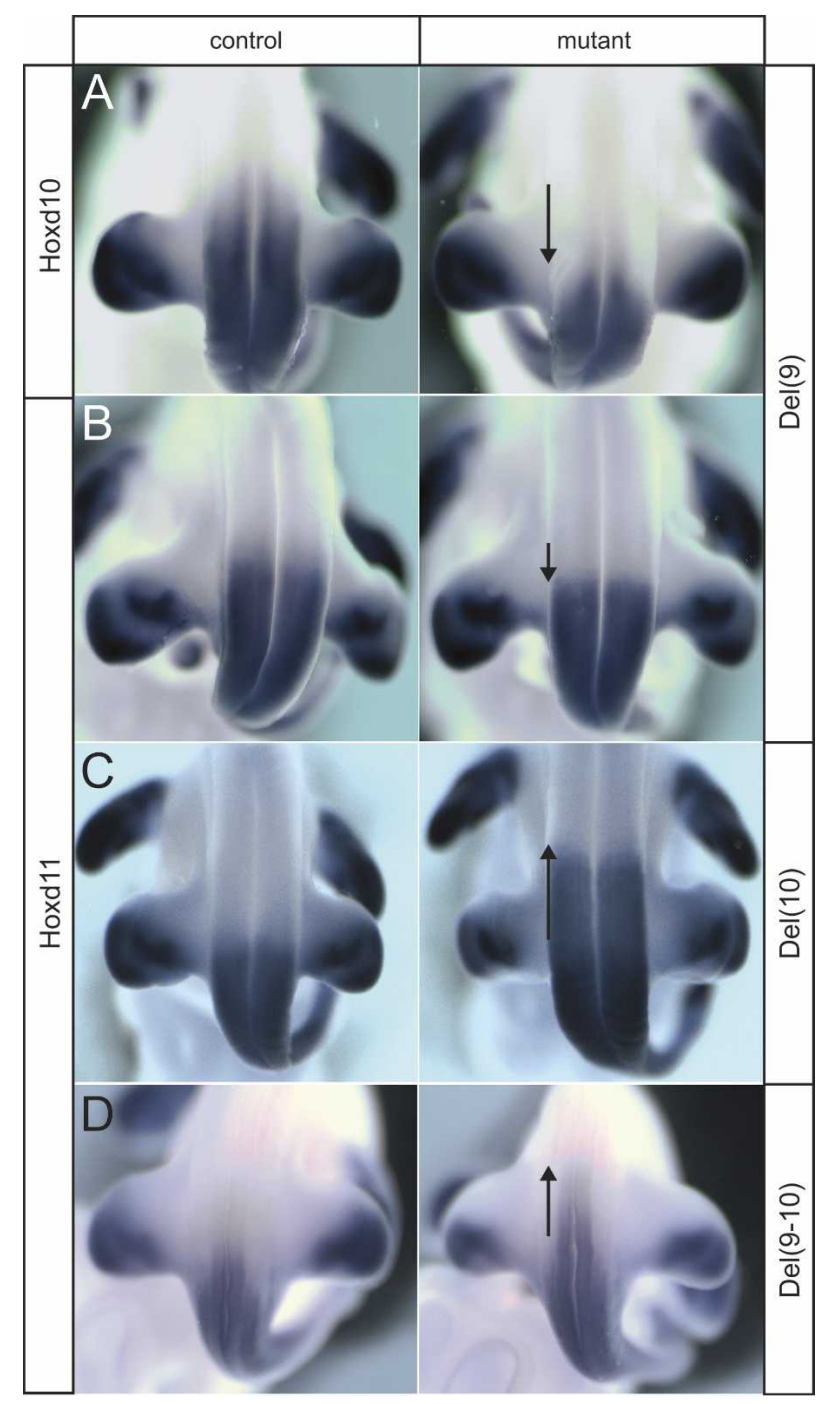

Figure 7. Spinal shifts in Hox gene expression in various deletion stocks. $(A, B)$ The lumbar homeosis observed in the Del(9) allele (Fig. 5E) correlates with significant caudal shifts in the expression of both Hoxd10 $(A)$ and Hoxd11 $(B)$ genes. Consequently, while only Hoxd9 is physically absent, the activities of Hoxd10 and Hoxd11 are also missing in the relevant lumbar region, leading to a severe anterior homeosis. $(C)$ Conversely, a rostral shift of Hoxd11 expression is scored in Del(10) animals, accounting for the unexpected posterior root transformation (Fig. 5B). (D) A comparable rostral shift of Hoxd11 expression is seen in Del(9-10). In this case, however, the additional loss of Hoxd9 function likely compensates for part of the Hoxd11 gain of function, generating a new functional balance with a normal nerve root organization (Fig. 5C).

intermediate intensity in the 21st-22nd DRG region, a somewhat lower intensity in the 23rd-24th region, whereas a stronger signal was detected around the 25th level (Fig. 9B-E).

As for Raldh2, group B deletions showing nerve root homeotic transformations displayed rostro-caudal shifts of Pea3-positive cells. For example, embryos lacking the HoxD cluster, with a partial anterior homeosis, dis- played a posterior shift of Pea3-positive MNs, most evident at the anterior lumbar level [Fig. 9B, Del(1-13)]. Conversely, posterior homeosis induced by the Hoxd11 lumbar gain of function in $\operatorname{Del}(10)$ was accompanied by an anterior shift of the caudal Pea3 pools [Fig. 9C, Del(10)]. In all cases, the extent of homeosis and motor pool shifts was coordinated and involved a maximum of one segment. Deletions without associated root homeosis [e.g., Del(8-10)] showed normal Pea3 patterns (data not shown). Nerve roots homeosis thus reflected the shifts of motor pools induced by modified Hoxd gene expression as suggested by the Lim1taulacZ experiments.

Group A deletions showed a substantial loss of Pea3positive cells at DRG levels 21-24, whereas the strong signal normally seen at the 25th level was shifted caudally in homozygous specimens [e.g., Del(10-13)] (Fig. 9D), the loss being already scored in Irn heterozygous animals (Fig. 9E). Because the latter animals otherwise maintained a normal nerve root pattern, we concluded that loss of Pea3 signal is caused by the Evx2 gain of function, rather than by alterations in Hoxd gene expression.

To further assess the fate of the LMC in our group A mutants, we immunostained neighbor sections of E14.5 Del(10-13) mutant animals with antibodies against HB9, whose robust expression labels the lateral subset of the LMC (LMCl) (Arber et al. 2000) and Islet1 (Is11), a marker of the medial subset of the LMC (LMCm) as well as of the MMC (Tsuchida et al. 1994). A third set of sections was stained for cleaved caspase-3, to detect potential variation in the number of apoptotic cells (Wang et al. 2001). At DRG levels 21-22, where Raldh2 and Pea3 expression was mostly decreased in this mutant stock, a virtually complete disappearance of the lateral-most Hb9 staining was observed, suggesting either the depletion or misidentification of the $\mathrm{LMCl}$ at this level (Supplementary Fig. S1A-C). However, apoptotic cells were not increased in number between E11.5 and E15.5 (Supplementary Fig. S1D-F). Since Evx2, as Evx1, is a post-mitotic marker for V0 interneurons, and because Hb9 inactivation leads to an increased number of $C h \times 10$ positive V2 interneurons, we assessed potential $\mathrm{MN}$ respecification by looking at Evx1 and Chx10 (Arber et al. 1999; Thaler et al. 1999; Moran-Rivard et al. 2001). No abnormal expression of these markers was detected between E10.5 and E11.5 in group A mutants (data not shown).

\section{Discussion}

\section{The mouse Irn mutation is allelic to HoxD}

In the spinal cord, loss of function of Hox genes has been associated with motor alterations at the level of both brachial and lumbo-sacral plexii, leading to detectable handicaps (Carpenter et al. 1997; Tiret et al. 1998). In these cases, abnormal innervation of the limbs was accounted for by a misidentification of the related motoneurons, perhaps resulting from spinal cord homeosis. 


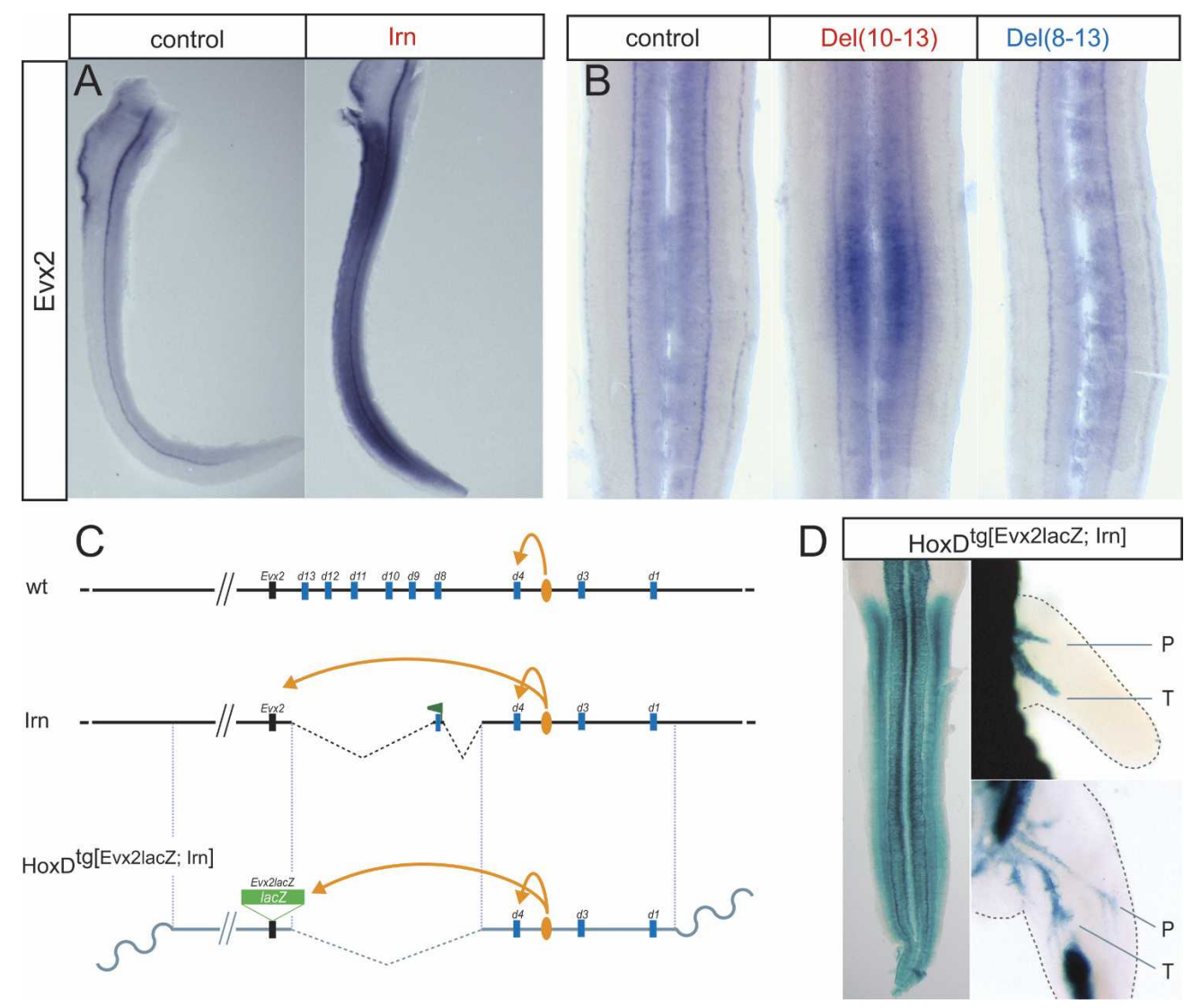

Figure 8. Complete leg paralysis in group A animals is associated with the gain of expression of Evx2 in lumbar LMC motoneurons. (A) E11.5 half-spinal cords of control (left) and Irn mutants (right). (Left) Expression of Evx2 is found in a column of interneurons. (Right) In the mutant stock, Evx2 is largely gained in a broad Hox-like spinal domain. (B) E12.5 LS spinal cords in an open-book configuration. All group A animals displayed a related Evx2 gain of expression, though limited to the ventral spinal cord at lumbar level [here Del(10-13)]. In contrast, less severely paralyzed (group B) animals sharing the same deletion breakpoint near Evx2 are devoid of ectopic Evx2 transcripts [e.g., Del(8-13)]. (C) In group A deletions, the Evx2 gene responds to neural regulatory sequences normally acting on Hoxd genes (yellow arrows). A modified HoxD BAC clone, with a deletion mimicking Irn outer breakpoints and a LacZ reporter cassette within Evx2 is used to generate transgenic mice (Hox $\left.D^{\operatorname{tg}\left[E_{v x} 21 a c Z ; I r n\right]}\right)$. ( $D$, left panel) X-gal staining of transgenic embryos confirms the impact of the deletion on Evx2 regulation (open-book view). Axon fibers innervating the hindlimb, but not the forelimb, are labeled, demonstrating that the spinal gain of expression includes lumbar LMC motoneurons (upper right and lower right panels, E11.5 and E13.5 hindlimbs, respectively). (T) Tibial nerve; (P) peroneal nerve.

Our characterization of both the mouse Ironside mutation and of several engineered deletions in the $\operatorname{Hox} D$ cluster shed some light on the molecular etiology(ies) of Hox-induced motor defects, which cannot be solely explained in terms of homeotic transformations.

Irn mutant mice were isolated due to a severe hindlimb paralysis affecting heterozygous animals. The fact that animals heterozygous for a complete HoxD deletion do not display such a locomotion problem (Zakany et al. 2001) raised questions about the molecular nature of this mutation. The characterization of Irn confirmed that the HoxD cluster had been modified, yet it did not solve the abovementioned paradox, which was reinforced by subsequent analysis of several engineered HoxD deletions also revealing paralyses more severe than those associated with a mere cluster deletion. This latter observation ruled out the presence, in Irn mice, of additional HoxDindependent genetic modifications adding to the phenotype. Likewise, potential implications of either the first exon of Hoxd8 or the 200-bp foreign fragment were ignored, as similar effects were obtained without such peculiarities. The Irn mutation was thus functionally considered as a simple large deletion removing from upstream of Hoxd4 to upstream of Hoxd13.

\section{Multiple factors induce distinct paralyses}

The analysis of both the Irn mutation and our deleted stocks revealed multifactorial causes for the various paralyses, involving at least three aspects. First, all affected strains lacked Hoxd10, making Hoxd10 inactivation mandatory for paralysis to occur. Second, a variety of either complete or incomplete nerve roots homeotic transformations was associated with the deleted configurations, which could impact on the organization of the lumbo-sacral plexus, leading to motor problems. Third, the strong paralysis observed in group A animals also involved a gain-of-function effect associated with the po- 
Figure 9. Dominant paralysis in group A specimens is associated with a massive loss of hindlimb LMC motoneurons, including Pea3-positive motor pools. $(A-E)$ E12.0-E13.0 LS spinal cords in an open-book configuration. Numbers refer to DRG levels. (A) At lumbar level, the LMC position is revealed by Raldh2 staining and appeared caudally shifted in both Del(10-13); $E v x 2^{\text {stop }}$ and Del(10-13) alleles. However, only the latter group A allele is accompanied by a significant loss of signal at levels 21-22, suggesting a loss of $\mathrm{MN}$ innervating the hindlimb. $(B, C)$ Group B alleles show Pea3 expression reminiscent of the nerve root homeotic transformations scored after neurofilament stainings, with some motor pools shifted either caudally in anterior root transformations $[\operatorname{Del}(1-13)](B)$ or rostrally in posterior transformations $[\operatorname{Del}(10)](C) .(D, E)$ More dramatic alterations of the $P e a 3$ expression domains are scored in group A mutants. Staining in the 21-24 region (arrowhead) is reduced in Del(10-13) homozygous, indicating that the corresponding motor pools are strongly depleted. A decrease in staining intensity is already visible in Irn heterozygotes in E. The Pea3-positive pools at level 25 appear normal (arrow), although slightly shifted caudally in Del(10-13) homozygous. Differences in staining intensities reflect differences in age, with E12.0 samples [Del/113), Irn] showing a stronger signal than E12.5E13.0 samples [Del(10), Del(10-13)].

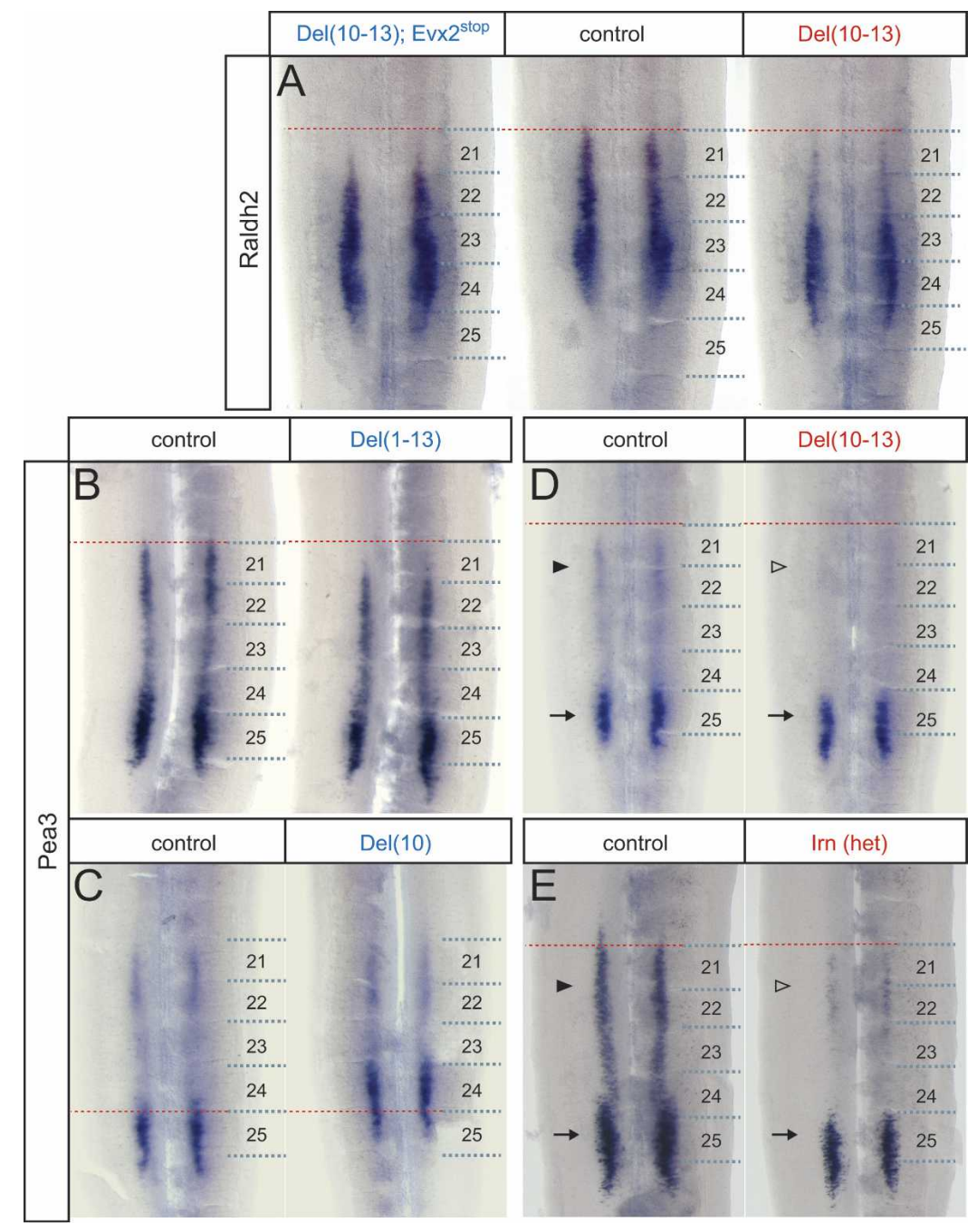

sition of the L1 breakpoint, since all group A deletions shared this 5'-extremity upstream of Hoxd13.

The loss of the embryonic peroneal nerve was occasionally observed in Hoxd10 mutant hindlimbs, yet most adults showed a normal leg innervation (Carpenter et al. 1997). In our hands, these latter fetuses displayed peroneal defects classified as "thinner/shorter." In contrast, a proportion of E12.5 group B animals were of the "truncated" category, generally leading to adults lacking this particular nerve. Altogether, our group B animals were thus more severely affected than the Hoxd10 mutant mice, which may result from the absence and/or deregulation of other Hoxd genes expressed at lumbosacral levels, an additive effect previously described for Hoxd10 and Hoxa10 (Wahba et al. 2001). However, the loss of the other Hoxd function has no effect as long as Hoxd10 was present, and Hoxa10 expression was found to be normal in group A embryos (data not shown). This and the fact that our group A animals are more affected than double group 10 mutants (Wahba et al. 2001) further suggest that additional alterations in gene activity are involved in the increased severity scored in group A animals.
While the extent of paralysis and the peroneal defects correlate well in the various alleles, other deficiencies participate in gait abnormality. Group B internal deletions, like Hoxd10 inactivation (Carpenter et al. 1997), led to outer foot rotation, and additional innervation defects occurred beside the reduction of the peroneal branch such as a dorso-lumbar plexus deprived of fibers in group A deletions.

\section{Nerve roots homeosis in HoxD deletions}

The position of both embryonic nerve roots and adult LMC in Hoxd10 mutants led Carpenter et al. (1997) to propose that alterations in $\mathrm{MN}$ positional identity are reflected by changes in peripheral innervation patterns; the 23rd nerve acquires a more anterior 22nd identity and ectopically projects to the lumbar plexus, thus reducing the number of axons projecting through the sacral plexus. In our hands, nerve root homeosis in Hoxd10 ${ }^{-/}$ animals was most prominent as partial anterior transformations affecting the 25th-27th nerves, even though we consistently noticed a slight reduction of the 23rd nerve contribution to the lumbar plexus. Yet the analysis of 
nerve roots in our mutants did not establish a firm correspondence between the type and/or level of homeotic transformations and the severity of the phenotype. For example, while reduced 23rd nerve contribution to the sacral plexus may lead to peroneal depletion, stronger peroneal phenotypes (and concomitant locomotion defects) were observed in the absence of homeosis, in some group B animals [e.g., Del(8-10)].

Conversely, homeotic transformations were scored in strains barely paralyzed, if at all [e.g., Del(9)]. In this case, it is unlikely that transformed 24th and 25th nerves could compensate for the redirection of some 23rd nerve fibers to the lumbar plexus, since the 24th root was systematically the most reluctant to anterior transformations. Altogether, nerve root homeosis was thus neither necessary, nor sufficient, for the emergence of a severely truncated peroneal branch. However, it could be instrumental in the occurrence of milder peroneal reductions, as for $\operatorname{Del}(9)$ embryos occasionally showing a thinner/ shorter peroneal nerve.

Nerve root homeosis at lumbar levels was nevertheless always found in group A animals with complete paralysis, even though it was also observed for all deletions lacking Hoxd10 and starting with the L1 breakpoint (including both A and B representatives). The Lim1taulacZ experiment suggests an explanation for this, as only group A alleles showed a robust and global lumbar motor homeosis. Therefore, anterior motor homeosis affecting the 23rd and 24th motor nerves might be critical for the loss of the dorso-sacral plexus and its emerging peroneal branch, whereas formation of this plexus is compatible with the converse posterior homeosis. We do not think that incorrect register between the limbs and the position of motoneurons participates in the phenotype, as the pelvic bone was found displaced accordingly. Also, complete anterior homeosis was described for Hoxa10-/adult mice, without any paralysis (Rijli et al. 1995), further documenting that full anterior homeosis is compatible with normal motor innervation and suggesting that peripheral innervation defects derive from partial, rather than complete transformations in nerve identities.

Our conditional deletion approach makes it unlikely that loss of the Hoxd gene in limb mesenchyme is involved in the paralysis. Instead, shifts in the position of lumbar LMC, revealed either by the Lim1taulac Z reporter or by the rostro-caudal location of Raldh2- and Pea3-positive cells, are likely critical in nerve roots homeosis. The primary cause of paralysis thus lies in the misidentification, wrong position, or disappearance of pools of motoneurons, consequent to modifications in the structure of the HoxD cluster, which in turn lead to various Hox losses and/or gains of function derived from the reallocation of enhancer activity. The analysis of gene expression in our various deletions confirmed this hypothesis.

\section{Ectopic expression of Evx2}

In group A animals, Evx2 adopted a Hox pattern up to various spinal cord rostral levels. Since all group A dele- tions share the same $5^{\prime}$-breakpoint, it is likely that the proximity of this breakpoint to the Evx2 promoter triggers this up-regulation. Interestingly, some deletions carrying the same breakpoint showed a less severe paralysis (group B). However, such deletions were either associated with the inactivation of Evx2 [Del(10-13); Evx2 $\left.{ }^{\text {stop }}\right]$ or showed normal Evx2 expression [Del(8-13)]. In the latter case, the presence of a boundary or insulator element in the intergenic region " $\mathrm{i}$ " likely prevented Evx2 misregulation, since further deletion of this region [in Irn or Del(i-13)] led to Evx2 up-regulation and consequent severe paralysis. Finally, the transgene replacing the HoxD cluster in Del(1-13) animals is silent in the spinal cord (Spitz et al. 2001), indicating the concomitant deletion of neural enhancers in this group $B$ allele, hence the failure to activate $E_{V x} 2$. Evx2 was never found deregulated in deletions internal to the HoxD cluster.

Our transgenic approach, whereby the Irn deletion was reproduced and generated a similar gain of Evx2 in the spinal cord, confirmed a role for the Hoxd4-Hoxd3 neural enhancers (Morrison et al. 1997; Zhang et al. 1997) in ectopic Evx2 expression. In these transgenic animals, the staining of hindlimb nerves by the Evx2lacZ reporter gene demonstrates misexpression within LMC neurons, supporting a detrimental function of EVX2 there. Likewise, the brachial nerves were not labeled in transgenic embryos, correlating with normal forelimb motility in Irn mutants.

\section{Lumbo-sacral motoneuron alterations}

The decrease in Raldh2 and Pea3 expression in our group A mutants indicated that some MN within the LMC were affected following ectopic expression of Evx2. The EVX2 protein labels V0 commissural interneurons (Moran-Rivard et al. 2001), which also express the paralogous gene Evx1 (Dollé et al. 1994). While the function of Evx2 in these cells is still elusive, Evx1 mutant mice display cell fate transformations consistent with a role as a determinant of IN identity (Moran-Rivard et al. 2001). Furthermore, the Evx1/2 Caenorhabditis elegans gene vab-7 (Esmaeili et al. 2002), while necessary for the determination of some motoneurons, can repress alternative $\mathrm{MN}$ fates, much in the same way as the Drosophila orthologous gene evenskipped (eve) (Landgraf et al. 1999; Broihier and Skeath 2002; Esmaeili et al. 2002; Odden et al. 2002). In both cases, pairs of homeodomain transcription factors specify differences between distinct $\mathrm{MN}$ identities through the inhibition of alternative fates, a mechanism also described in the specification of progenitor domains in the vertebrate neural tube (Jessell 2000).

In vertebrates, the $\mathrm{MN}$-specific homeodomain factor Hb9 can suppress interneuron specification (Arber et al. 1999; Thaler et al. 1999), suggesting that the converse influence may also occur. The quasidisappearance of strong $\mathrm{Hb}$ 9-positive cells in the $\mathrm{LMCl}$ of group A mutants suggests that the antagonism between eve and Hb9, documented in Drosophila, is retained in vertebrates such that ectopic EVX product interferes with the 
identification and/or fate of $\mathrm{LMCl}$ neurons. In this respect, it is noteworthy that the mouse EVX1 product can rescue eve mutant phenotype in the Drosophila nervous system, where abnormal MN specification coincides with the derepression of $H b 9$ (Fujioka et al. 2003). Whether the loss of $\mathrm{Hb} 9$ signal reflects an absence of $\mathrm{LMCl}$ is as yet unclear. At anterior LMC levels, the detection of the Lim1 signal (specific for LMCl) on emerging axons suggests that at least some LMCl MNs are present, whereas the absence of dorso-sacral plexus at more posterior levels suggests the opposite.

\section{Misspecification of motoneuron pools}

While the fate of group A LMCl cells normally expressing $\mathrm{Hb} 9$ is unknown, they likely do not enter apoptosis, as the overall number of apoptotic cells in this region was not significantly changed. Also, a shift of $\mathrm{LMCl}$ cells toward a V0 or V2 interneuron identity is unlikely, when considering the normal EvX1 and Chx10 expression in mutant spinal cord.

Pea3-positive lumbar pools do not contain MN innervating the distal limb compartment, either via the tibial or the peroneal nerves. Instead, they correspond to $\mathrm{MN}$ innervating more proximal limb targets (McHanwell and Biscoe 1981; Arber et al. 2000). The absence of Pea3 signal observed in group A animals thus points to an innervation deficit in proximal hindlimb. This may explain the full leg paralysis observed in these specimens, as opposed to the distally restricted clubfoot phenotype of group B representatives, as the mere loss of the peroneal nerve is not expected to produce complete leg paralysis. Accordingly, in EphA4 mutant mice, the lack of peroneal nerves due to the redirection of LMC projection toward the ventral limb mesenchyme leads to a limited clubfoot phenotype (Helmbacher et al. 2000). While this hypothesis explains the increased severity of group A deletions through an additive effect, its demonstration is made difficult by the absence of markers expressed in peroneal motor pools, which should then be defective in both group A and B mutants. Interestingly, more MN express $P e a 3$ at brachial versus lumbar levels, and a subtle defect in forelimb motor coordination was reported following Pea3 inactivation (Livet et al. 2002).

We conclude that the two categories of paralyzed phenotypes reported here are likely produced by at least two different molecular mechanisms. The first one relies on the loss of Hoxd10 function, which impacts mildly on the peroneal nerve branch, leading to a distal limb phenotype in both group A and B animals. The second factor is a gain of function of $E_{v \times 2}$, which alters the determination of $\mathrm{MN}$ pools specific for more proximal innervation, thus leading to a full leg paralysis in group A animals only. The potential causal role of homeotic transformations in these phenotypes is more difficult to assess, as no strict correlation was observed between homeosis and the phenotypes. However, severely affected animals all had partial, rather than complete, transformations in their nerve roots patterns, indicating that an internal pattern disorganization is more detrimental than a complete shift.

\section{Materials and methods}

Isolation and mapping of the Ironside (Irn) spontaneous mutation

The Irn mutation was identified at The Jackson Laboratory, Maine, USA, from a single mutational event in an NZBW F1 mouse specimen showing severe hindlimb defects. In a cross with $\mathrm{CAST} / \mathrm{EiJ}$ and $\mathrm{C} 3 \mathrm{HeB} / \mathrm{FeJ}$ strains, the region containing the mutation was narrowed to a 6-cM interval between markers D2Mit10 and D2Mit418 on mouse chromosome 2. As this mutation first appeared in F1 hybrid mice, polymorphic markers were used to establish that the mutation originated from the NZW/LacJ male parental chromosome and not the female NZB/ $\mathrm{B} 1 \mathrm{NJ}$ parental chromosome. The strain is currently maintained at The Jackson Laboratory on a B6C3Fe hybrid background. Breeding heterozygous animals generated mice with more severe hindlimb paralysis as well as digit defects. Southern blot and PCR analysis revealed that the mutation consisted in a complex deletion/insertion event in the HoxD cluster and allowed to further clone and sequence the breakpoints.

LoxP mouse strains and targeted meiotic recombination (TAMERE)

Two distinct parental strains carrying a $\operatorname{Lox} P$ site at the L1 position (Fig. 2) were used to produce genomic rearrangements in vivo. One strain contained a mutation within the Evx2 gene (Hérault et al. 1996) and was used to produce the Del(10-13); $E_{V x} 2^{\text {stop }}$ allele. All other deletions with a L1 breakpoint were obtained via another LoxP strain associated to a short deletion removing a conserved intergenic region upstream of Evx2 (Kmita et al. 2002). For the sake of clarity, and because this intergenic sequence did not interfere either with the phenotypes or with the regulatory effects reported here, it is not mentioned in Figure 2. The L4 strain was already described (Gérard et al. 1996). The L5, L6, L7, and L8 strains were produced by routine ES cell technology, and carry a single LoxP site at the following positions: between Hoxd10 and Hoxd9 (L5: AAGGAGA TGGGAGGA *CTGGAGAAGACCTAG), between Hoxd9 and Hoxd8 (L6: TTTCCGGACAGGCGC^TCGAGATGTAGCAAA), 3' to Hoxd8 (L7: GCACTTACGTGCATG ${ }^{\star}$ CTGCCAAAACA $^{\prime}$ TTTG), and 5' to Hoxd4 (L8: GACAGCTCTCCTCTA*AGCT TCTCTACCAAC). The various crosses to obtain targeted meiotic recombination events from two distinct LoxP parental strains, in the presence of the Sycp1-Cre transgene, are like those described elsewhere (Hérault et al. 1998). Animals heterozygous for a given deletion event were recovered with a frequency of $0.1 \%-5 \%$.

The Flox(4-13) chromosome was produced by two rounds of targeting in ES cells as already described for the corresponding deletion (Zakany and Duboule 1999); the line used here corresponds to the germline transmission of the floxed clone before Cre treatment in vitro. The L11 position corresponds to a $L o x P$ targeting event used to produce a deletion of the entire HoxD cluster in ES cells (Zakany et al. 2001). The Lim1taulacZ mouse strain was provided by A. Kania and T. Jessell (Howard Hughes Medical Institute, Columbia University, New York), and the Hoxd10 insertional mutant stock by E. Carpenter (University of California at Los Angeles School of Medicine, Los Angeles, CA).

HoxD BAC recombination and transgenesis

A mouse BAC (RPCI23-400H17) carrying the entire HoxD complex and $\sim 100 \mathrm{~kb}$ of centromeric sequence (Spitz et al. 2001) was 
used to generate a large deletion removing the Hoxd13 to Hoxd8 genes. A kanamycine resistance gene was amplified by PCR using primers bearing $\sim 50-\mathrm{pb}$ homology to the genomic sequences located on each side of the intended deletion at their $5^{\prime}$ extremities $\left(5^{\prime}\right.$ deletion/ $\mathrm{Kan}^{R}$ : CGCGACACCTTCATTCATT AGTGGTTTTGAAACTACCTTGCACCTTTTAAGTAACAT CGGATCCTAGTAAGCCACG; 3' deletion/ $\mathrm{Kan}^{R}$ : CCACCCA AGAAGAAACCAAGGCCAGGACATTGGCTCCCCAGTTC TATAAATAGAAGTCCCCTACCCGGTAGAATTC). Sequence homology was selected such that the engineered breakpoints on the BAC exactly matched the outer breakpoints mapped in the Irn mutant. Upon bacterial electroporation, the resulting PCR product thus replaced $50 \mathrm{~kb}$ of $400 \mathrm{H} 17 \mathrm{BAC}$ sequence (including the Hoxd13 to Hoxd8 genes) by the $\mathrm{Kan}^{R}$ selection cassette. To monitor Evx2 gene expression from the deleted $400 \mathrm{H} 17$ BAC, the LacZ coding sequence was inserted in frame such as to disrupt the homeodomain located in the second exon. Primers bearing convenient restriction sites and 50-pb homology to the Evx2 homeodomain at their 5' extremities were used to amplify a plasmid backbone containing the R6K origin of replication (BamHI/3'homeo/R6K: GGCGGATCCTACAGGGAGAACTA CGTGTCTCGGCCCCGCCGGTGCGAGCTGGCCGCTGCA CTCACCCCTCTTTAATACGACGGGC; Sall/5'homeo/KpnI/ R6K: ATCGTCGACCGAACTCTTTCTCCAGGCGCGCGAT CTGTTCGCGGGTGAATGCCGTACGGTATCGCGGTACC CTGTGAATGCGCAAAC). Following the conventional cloning of a LacZ-zeocin ${ }^{\mathrm{R}}$ cassette in this customized plasmid backbone, the insert carrying the LacZ-zeocin ${ }^{\mathrm{R}}$ flanked by the two homology arms was excised and electroporated into bacterial cells containing the deleted $400 \mathrm{H} 17$ BAC. After linearization, the modified BAC was introduced in C57BL/6XDBA F1 fertilized mouse eggs by pronuclear injection.

Whole-mount in situ hybridization (WISH), immunochemistry, and X-gal stainings

WISH was carried out on fetuses between 11.5 and $12.5 \mathrm{~d}$, using standard procedures and previously described probes. In several cases, the spinal cord was opened before hybridization for better probe accessibility. After staining, entire or half-spinal cords were dissected out and mounted in PBS/glycerol under a coverslip. Entire spinal cords are presented in an open-book configuration, viewed from a ventral aspect (Livet et al. 2002). Wholemount neurofilament immunostainings were carried out on fetuses between 11.5 and $13.5 \mathrm{~d}$, using standard procedures. The antibodies were anti-NF160 (clone NN18, Sigma N-5264) and anti-mouse Ig Fab HRP conjugate (Sigma A-3882). After antibody incubation, the embryos were stained with DAB substrate and cleared by dehydration in methanol, followed by incubation in BABB (1:2 benzyl alcohol/benzyl benzoate). Following X-gal detection of LacZ activity in BAC transgenic and Lim1taulacZ experiments, the embryos were cleared by the same procedure.

\section{Immunohistochemistry}

The antibodies used were rabbit anti-Isl1 (A8; A. Pierani [Ecole Normale Superieure, Paris, France] and T. Jessell), rabbit anti$\mathrm{Hb} 9$ (S. Arber [Biozentrum, University of Basel, Basel, Switzerland]), and rabbit anti-cleaved caspase-3 (Asp 175) for detection of apoptotic cells (Cell Signaling Technology).

\section{Leg footprints}

Adult mice were encouraged to run on a light-sensitive film after having their hindfeet soaked in developer solution. After processing, exposed regions correspond to footprints.

\section{Acknowledgments}

We thank S. Arber, T. Jessel, and A. Pierani for probes and antibodies; A. Kania, T. Jessell, E. Carpenter, M. Logan, M. Kmita, and J. Zakany for mice; and S. Arber and J. Zakany for discussions and suggestions. This work was supported by funds from the canton de Genève, the Claraz and Louis Jeantet Foundations, the Swiss National Research Fund, the National Research Centre (NCCR) "Frontiers in Genetics," and the EU programmes "EUMORPHIA" and "Cells into Organs."

\section{References}

Arber, S., Han, B., Mendelsohn, M., Smith, M., Jessell, T.M., and Sockanathan, S. 1999. Requirement for the homeobox gene $\mathrm{Hb} 9$ in the consolidation of motor neuron identity. Neuron 23: 659-674.

Arber, S., Ladle, D.R., Lin, J.H., Frank, E., and Jessell, T.M. 2000. ETS gene Er81 controls the formation of functional connections between group Ia sensory afferents and motor neurons. Cell 101: 485-498.

Broihier, H.T. and Skeath, J.B. 2002. Drosophila homeodomain protein $\mathrm{dHb} 9$ directs neuronal fate via crossrepressive and cell-nonautonomous mechanisms. Neuron 35: 39-50.

Carpenter, E.M. 2002. Hox genes and spinal cord development. Dev. Neurosci. 24: 24-34.

Carpenter, E.M., Goddard, J.M., Davis, A.P., Nguyen, T.P., and Capecchi, M.R. 1997. Targeted disruption of Hoxd-10 affects mouse hindlimb development. Development 124: 4505-4514.

Dasen, J.S., Liu, J.P., and Jessell, T.M. 2003. Motor neuron columnar fate imposed by sequential phases of Hox-c activity. Nature 425: 926-933.

Davenne, M., Maconochie, M.K., Neun, R., Pattyn, A., Chambon, P., Krumlauf, R., and Rijli, F.M. 1999. Hoxa2 and Hoxb2 control dorsoventral patterns of neuronal development in the rostral hindbrain. Neuron 22: 677-691.

Dollé, P., Izpisua-Belmonte, J.C., Brown, J.M., Tickle, C., and Duboule, D. 1991. HOX-4 genes and the morphogenesis of mammalian genitalia. Genes \& Dev. 5: 1767-1776.

Dollé, P., Dierich, A., LeMeur, M., Schimmang, T., Schuhbaur, B., Chambon, P., and Duboule, D. 1993. Disruption of the Hoxd-13 gene induces localized heterochrony leading to mice with neotenic limbs. Cell 75: 431-441.

Dollé, P., Fraulob, V., and Duboule, D. 1994. Developmental expression of the mouse Evx-2 gene: Relationship with the evolution of the HOM/Hox complex. Development Suppl: 143-153.

Duboule, D. and Morata, G. 1994. Colinearity and functional hierarchy among genes of the homeotic complexes. Trends Genet. 10: 358-364.

Esmaeili, B., Ross, J.M., Neades, C., Miller III, D.M., and Ahringer, J. 2002. The C. elegans even-skipped homologue, vab7, specifies DB motoneurone identity and axon trajectory. Development 129: 853-862.

Fujioka, M., Lear, B.C., Landgraf, M., Yusibova, G.L., Zhou, J., Riley, K.M., Patel, N.H., and Jaynes, J.B. 2003. Even-skipped, acting as a repressor, regulates axonal projections in Drosophila. Development 130: 5385-5400.

Gaunt, S.J., Sharpe, P.T., and Duboule, D. 1988. Spatially restricted domains of homeogene transcripts in mouse embryos: Relation to a segmented body plan. Development Supp. 104: 169-180.

Gérard, M., Chen, J.Y., Gronemeyer, H., Chambon, P., Duboule, D., and Zakany, J. 1996. In vivo targeted mutagenesis of a regulatory element required for positioning the Hoxd-11 and 
Hoxd-10 expression boundaries. Genes \& Dev. 10: 23262334.

Graham, A., Maden, M., and Krumlauf, R. 1991. The murine Hox-2 genes display dynamic dorsoventral patterns of expression during central nervous system development. Development 112: 255-264.

Helmbacher, F., Schneider-Maunoury, S., Topilko, P., Tiret, L., and Charnay, P. 2000. Targeting of the EphA4 tyrosine kinase receptor affects dorsal/ventral pathfinding of limb motor axons. Development 127: 3313-3324.

Hérault, Y., Hraba-Renevey, S., van der Hoeven, F., and Duboule, D. 1996. Function of the Evx-2 gene in the morphogenesis of vertebrate limbs. EMBO J. 15: 6727-6738.

Hérault, Y., Rassoulzadegan, M., Cuzin, F., and Duboule, D. 1998. Engineering chromosomes in mice through targeted meiotic recombination (TAMERE). Nat. Genet. 20: 381-384.

Jessell, T.M. 2000. Neuronal specification in the spinal cord: Inductive signals and transcriptional codes. Nat. Rev. Genet. 1: 20-29.

Kania, A., Johnson, R.L., and Jessell, T.M. 2000. Coordinate roles for LIM homeobox genes in directing the dorsoventral trajectory of motor axons in the vertebrate limb. Cell 102: 161-173.

Kmita, M. and Duboule, D. 2003. Organizing axes in time and space; 25 years of colinear tinkering. Science 301: 331-333.

Kmita, M., Tarchini, B., Duboule, D., and Herault, Y. 2002. Evolutionary conserved sequences are required for the insulation of the vertebrate Hoxd complex in neural cells. Development 129: 5521-5528.

Landgraf, M., Roy, S., Prokop, A., VijayRaghavan, K., and Bate, M. 1999. even-skipped determines the dorsal growth of motor axons in Drosophila. Neuron 22: 43-52.

Livet, J., Sigrist, M., Stroebel, S., De Paola, V., Price, S.R., Henderson, C.E., Jessell, T.M., and Arber, S. 2002. ETS gene Pea3 controls the central position and terminal arborization of specific motor neuron pools. Neuron 35: 877-892.

Logan, M., Martin, J.F., Nagy, A., Lobe, C., Olson, E.N., and Tabin, C.J. 2002. Expression of Cre Recombinase in the developing mouse limb bud driven by a Prxl enhancer. Genesis 33: $77-80$.

Lumsden, A. and Krumlauf, R. 1996. Patterning the vertebrate neuraxis. Science 274: 1109-1115.

McGinnis, W. and Krumlauf, R. 1992. Homeobox genes and axial patterning. Cell 68: 283-302.

McHanwell, S. and Biscoe, T.J. 1981. The localization of motoneurons supplying the hindlimb muscles of the mouse. Philos. Trans. R Soc. Lond. B Biol. Sci. 293: 477-508.

Moran-Rivard, L., Kagawa, T., Saueressig, H., Gross, M.K., Burrill, J., and Goulding, M. 2001. Evx1 is a postmitotic determinant of v0 interneuron identity in the spinal cord. Neuron 29: 385-399.

Morrison, A., Ariza-McNaughton, L., Gould, A., Featherstone, M., and Krumlauf, R. 1997. HOXD4 and regulation of the group 4 paralog genes. Development 124: 3135-3146.

Odden, J.P., Holbrook, S., and Doe, C.Q. 2002. Drosophila HB9 is expressed in a subset of motoneurons and interneurons, where it regulates gene expression and axon pathfinding. $J$. Neurosci. 22: 9143-9149.

Rijli, F.M., Matyas, R., Pellegrini, M., Dierich, A., Gruss, P., Dolle, P., and Chambon, P. 1995. Cryptorchidism and homeotic transformations of spinal nerves and vertebrae in Hoxa-10 mutant mice. Proc. Natl. Acad. Sci. 92: 8185-8189.

Shah, V., Drill, E., and Lance-Jones, C. 2004. Ectopic expression of Hoxd10 in thoracic spinal segments induces motoneurons with a lumbosacral molecular profile and axon projections to the limb. Dev. Dyn. 231: 43-56.

Sockanathan, S. and Jessell, T.M. 1998. Motor neuron-derived retinoid signaling specifies the subtype identity of spinal motor neurons. Cell 94: 503-514.

Spitz, F., Gonzalez, F., Peichel, C., Vogt, T.F., Duboule, D., and Zakany, J. 2001. Large scale transgenic and cluster deletion analysis of the HoxD complex separate an ancestral regulatory module from evolutionary innovations. Genes \& Dev. 15: 2209-2214.

Spitz, F., Gonzalez, F., and Duboule, D. 2003. A global control region defines a chromosomal regulatory landscape containing the HoxD cluster. Cell 113: 405-417.

Thaler, J., Harrison, K., Sharma, K., Lettieri, K., Kehrl, J., and Pfaff, S.L. 1999. Active suppression of interneuron programs within developing motor neurons revealed by analysis of homeodomain factor HB9. Neuron 23: 675-687.

Tiret, L., Le Mouellic, H., Maury, M., and Brulet, P. 1998. Increased apoptosis of motoneurons and altered somatotopic maps in the brachial spinal cord of Hoxc-8-deficient mice. Development 125: 279-291.

Trainor, P.A. and Krumlauf, R. 2001. Hox genes, neural crest cells and branchial arch patterning. Curr. Opin. Cell Biol. 13: 698-705.

Tsuchida, T., Ensini, M., Morton, S.B., Baldassare, M., Edlund, T., Jessell, T.M., and Pfaff, S.L. 1994. Topographic organization of embryonic motor neurons defined by expression of LIM homeobox genes. Cell 79: 957-970.

Wahba, G.M., Hostikka, S.L., and Carpenter, E.M. 2001. The paralogous Hox genes Hoxa10 and Hoxd10 interact to pattern the mouse hindlimb peripheral nervous system and skeleton. Dev. Biol. 231: 87-102.

Wang, J., Silva, J.P., Gustafsson, C.M., Rustin, P., and Larsson, N.G. 2001. Increased in vivo apoptosis in cells lacking mitochondrial DNA gene expression. Proc. Natl. Acad. Sci. 98: 4038-4043.

Yokouchi, Y., Sakiyama, J., and Kuroiwa, A. 1995. Coordinated expression of Abd-B subfamily genes of the HoxA cluster in the developing digestive tract of chick embryo. Dev. Biol. 169: 76-89.

Zakany, J. and Duboule, D. 1999. Hoxd genes and the making of sphincters. Nature 401: 761-762.

Zakany, J., Kmita, M., Alarcon, P., de la Pompa, J., and Duboule, D. 2001. Localized and transient transcription of Hox genes suggests a link between patterning and the segmentation clock. Cell 106: 207-217.

Zhang, F., Popperl, H., Morrison, A., Kovacs, E.N., Prideaux, V., Schwarz, L., Krumlauf, R., Rossant, J., and Featherstone, M.S. 1997. Elements both $5^{\prime}$ and $3^{\prime}$ to the murine Hoxd4 gene establish anterior borders of expression in mesoderm and neurectoderm. Mech. Dev. 67: 49-58. 


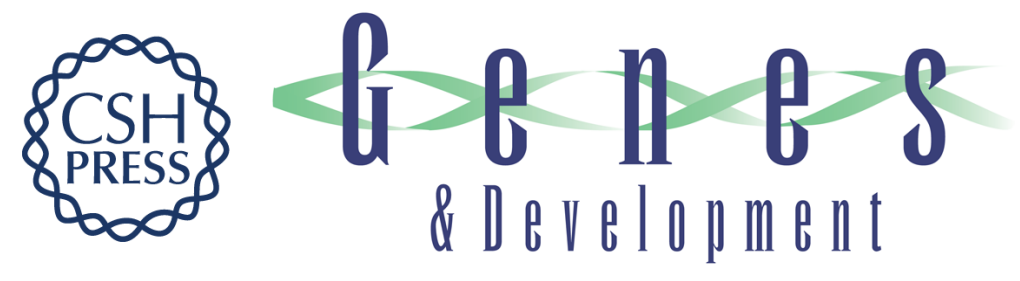

\section{HoxD cluster scanning deletions identify multiple defects leading to paralysis in the mouse mutant Ironside}

Basile Tarchini, Thi Hanh Nguyen Huynh, Greg A. Cox, et al.

Genes Dev. 2005, 19:

Access the most recent version at doi:10.1101/gad.351105

Supplemental
Material http://genesdev.cshlp.org/content/suppl/2005/11/15/19.23.2862.DC1

References This article cites 46 articles, 16 of which can be accessed free at:

http://genesdev.cshlp.org/content/19/23/2862.full.html\#ref-list-1

License

Email Alerting

Receive free email alerts when new articles cite this article - sign up in the box at the top

Service

right corner of the article or click here.

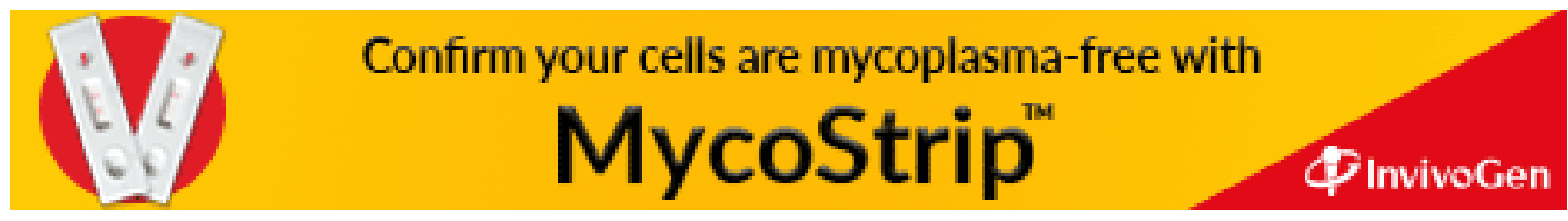

